\title{
Hippocampal Synapses Depend on Hippocampal Estrogen Synthesis
}

\author{
Oliver Kretz, ${ }^{1 \star}$ Lars Fester, ${ }^{2 \star}$ Uwe Wehrenberg, ${ }^{2}$ Lepu Zhou, ${ }^{2}$ Silke Brauckmann, ${ }^{1}$ Shanting Zhao, ${ }^{1}$ Janine Prange-Kiel, ${ }^{2}$ \\ Thomas Naumann, ${ }^{1}$ Hubertus Jarry, ${ }^{3}$ Michael Frotscher, ${ }^{1}$ and Gabriele M. Rune ${ }^{2}$ \\ ${ }^{1}$ Institute of Anatomy and Cell Biology, Albert-Ludwigs-Universität Freiburg, D-79104 Freiburg, Germany, ${ }^{2}$ Institute of Anatomy: Cellular Neurobiology, \\ Universitätsklinikum Hamburg-Eppendorf, D-20246 Hamburg, Germany, and ${ }^{3}$ Department of Obstetrics and Gynecology, University of Göttingen, D-37075 \\ Göttingen, Germany
}

Estrogens have been described to induce synaptogenesis in principal neurons of the hippocampus and have been shown to be synthesized and released by exactly these neurons. Here, we have focused on the significance of local estrogen synthesis on spine synapse formation and the synthesis of synaptic proteins. To this end, we reduced hippocampal estrogen synthesis in vitro with letrozole, a reversible nonsteroidal aromatase inhibitor. In hippocampal slice cultures, letrozole treatment resulted in a dose-dependent decrease of $17 \beta$ estradiol as quantified by RIA. This was accompanied by a significant decrease in the density of spine synapses and in the number of presynaptic boutons. Quantitative immunohistochemistry revealed a downregulation of spinophilin, a marker of dendritic spines, and synaptophysin, a protein of presynaptic vesicles, in response to letrozole. Surprisingly, no increase in the density of spines, boutons, and synapses and in spinophilin expression was seen after application of estradiol to the medium of cultures that had not been treated with letrozole. However, synaptophysin expression was upregulated under these conditions. Our results point to an essential role of endogenous hippocampal estrogen synthesis in the maintenance of hippocampal spine synapses.

Key words: estrogen; aromatase; letrozole; synaptic plasticity; synaptogenesis; spines

\section{Introduction}

Previous studies have shown an increased dendritic spine density in hippocampal CA1 pyramidal cells after estrogen treatment of ovariectomized rats and of hippocampal cell cultures (for review, see Segal and Murphy, 2001; McEwen, 2002). For the presynaptic side, a corresponding increase in the frequency of multiple synapse boutons in CA1 after estradiol application was described (Woolley et al., 1996; Yankova et al., 2001). Consistent with these findings, Crispino et al. (1999) reported changes in synaptic proteins after estrogen treatment. Likewise, an upregulation of synaptophysin and spinophilin immunoreactivity in response to estrogen was shown. Most of these effects could be blocked by estrogen receptor (ER) antagonists (Brake et al., 2001; Rune et al., 2002; Hao et al., 2003; Yokomaku et al., 2003).

Actions of estrogens are classically mediated by their binding to nuclear receptor subtypes, which results in binding of the receptor to an estrogen-responsive element and finally the expression of estrogen target genes (for review, see Klinge, 2001). Posttranscriptional effects, however, have also been described (L'Horset et al., 1990; Ing et al., 1996; Srivastava et al., 1997; Ing

Received May 26, 2003; revised April 28, 2004; accepted May 15, 2004.

This study was supported by the Deutsche Forschungsgemeinschaft (Ru 436/4-1 to G.M.R.; Fr 620/8-1 to M.F.). We thank Dr. J. Schulte-Mönting for statistical analysis of the data.

${ }^{*} 0$.K. and L.F. contributed equally to this work.

Correspondence should be addressed to Dr. Gabriele M. Rune, Zentrum für Experimentelle Medizin, Institut für Anatomie I: Zelluläre Neurobiologie, Universitätsklinikum Hamburg-Eppendorf, Martinistrasse 52, D-20246 Hamburg, Germany.E-mail: rune@uke.uni-hamburg.de.

DOI:10.1523/JNEUROSCI.5186-03.2004

Copyright $\odot 2004$ Society for Neuroscience $\quad$ 0270-6474/04/245913-09\$15.00/0 and Ott, 1999; Akama and McEwen, 2003; Yokomaku et al., 2003). In addition, estrogen effects are presumed to be mediated by putative, as yet not identified, membrane-bound receptors (Moss et al., 1997; Revelli, 1998; Razandi et al., 1999; ToranAllerand, 2002). This would imply that estrogens share common signal transduction pathways with other signal molecules (e.g., neurotrophic factors) (Linford et al., 2000; Balthazart et al., 2001; Bi et al., 2001; Björnström and Sjöberg, 2002; Shingo and Kito, 2002). In fact, such a cross talk between estrogens and neurotrophins has been documented (for review, see Toran-Allerand et al., 1999; Toran-Allerand, 2000).

In the present study, we attempted to downregulate hippocampal estrogen synthesis by using a reversible nonsteroidal aromatase inhibitor (letrozole). In the hippocampus, all enzymes of steroidogenesis are expressed, including aromatase, which converts testosterone to estradiol and represents the final step of estrogen synthesis (for review, see Azcoitia et al., 1999; Compagnone and Mellon, 2000; Garcia-Segura et al., 2001; Wehrenberg et al., 2001). A sixfold higher concentration of estradiol in the hippocampus than in plasma strongly indicates that estrogen synthesis takes place in the hippocampus (Hojo et al., 2004). Recently we have shown, for the first time, that adult hippocampal neurons indeed synthesize estrogens in vitro and that this synthesis is strongly attenuated by the inhibition of aromatase activity. Furthermore, these hippocampus-derived estrogens regulate the ER expression in hippocampal neurons and thereby indicate an auto/paracrine way of action (Prange-Kiel et al., 2003). Reversible nonsteroidal aromatase inhibitors actively de- 
crease the amount of circulating estrogens rather than eliminate the enzyme as known for irreversible steroidal agents. Anti-estrogens commonly used to abolish estrogen action by blocking ERs were shown to also exert agonistic effects (Nilsson and Gustafsson, 2002). Letrozole treatment, in contrast, results in very low levels of residual active aromatase (Höffken, 1999; Mitropoulou et al., 2003). Thus, we sought to remove the source of estrogen rather than neutralize its action by using ER antagonists.

We show that inhibition of estrogen synthesis by inactivating aromatase in hippocampal cultures reduces the density of spines and synapses and downregulates synaptic proteins. Unexpectedly, exogenous application of estradiol per se did not induce an increase in spines and synapses in culture. Our results point to a crucial role of endogenous hippocampal estrogen synthesis in controlling hippocampal synapse number.

\section{Materials and Methods}

Animals. Wistar rats (Institute of Anatomy and Cell Biology, University of Freiburg, Freiburg, Germany; Institute of Anatomy I: Cellular Neurobiology, University of Hamburg, Hamburg, Germany) were maintained under controlled conditions, and water and food were available ad libitum. Young postnatal rats ( $4-7 \mathrm{~d}$ of age; $n=64$ ) were anesthetized and decapitated for slice culture preparation or for cultivation of cells after dispersion. All experiments were performed in accordance with institutional guidelines for animal welfare.

Hippocampal slice cultures. Slice cultures were prepared as described in detail previously (Frotscher et al., 1995). Slices (400 $\mu \mathrm{m})$ of hippocampi from newborn rats were prepared as described and then cultivated according to the method introduced by Stoppini et al. (1991). Briefly, sections were stored at $4^{\circ} \mathrm{C}$ in a preparation solution (MEM and $2 \mathrm{~mm}$ glutamine) for 30-60 min. The sections were then placed on moistened translucent membranes $(0.4 \mu \mathrm{m}$ Culture Plate Insert, $30 \mathrm{~mm}$ diameter, Millicell-CM; Millipore, Eschborn, Germany), which were inserted in 6-well plates ( $35 \mathrm{~mm}$ in diameter) filled with $0.8 \mathrm{ml}$ of medium $(50 \%$ MEM, 25\% HBSS, and 25\% heat inactivated horse serum) with a final concentration of $2 \mathrm{~mm}$ glutamine and $0.044 \% \mathrm{NaHCO}_{3}$. The $\mathrm{pH}$ was adjusted to 7.3. The cultures were kept in vitro for $8 \mathrm{~d}$ at $37^{\circ} \mathrm{C}$ in a humidified, $\mathrm{CO}_{2}$-enriched atmosphere. The culture medium was changed three times per week.

Dispersion cultures. Cell culture preparation was performed as described by Brewer (1997), with slight modifications (Prange-Kiel et al., 2003). Briefly, meninges and white matter were removed from the prepared hippocampi; the tissue was minced and subsequently resuspended in digestion solution $\left(1.5 \mathrm{ml} /\right.$ hippocampus; $\left.30 \mathrm{~min} ; 30^{\circ} \mathrm{C}\right)$. Nondigested tissue was removed by filtration. The cell suspension was applied to the top of a gradient of Optiprep (Nycomed, Oslo, Norway). Optiprep was diluted in preparation medium (1:2), with a gradient consisting of four 1 $\mathrm{ml}$ steps of $35,25,20$, and $15 \%$ prediluted Optiprep in preparation medium. The suspension was centrifuged for $15 \mathrm{~min}$ at $800 \times g$ (Megafuge 1.0 R; Heraeus, Osterode, Germany). Cells collected in the lower two fractions were washed twice with preparation medium and then resuspended in culture medium and plated on poly-D-lysine $\left(20 \mu \mathrm{g} / \mathrm{cm}^{2}\right.$; Sigma, Deisenhofen, Germany)-coated glass tiles in 24-well culture dishes (diameter, $8 \mathrm{~mm}$; Nunc, Wiesbaden, Germany) at a density of $5 \times 10^{4}$ cells $/ \mathrm{ml} ; 1 \mathrm{ml}$ cell suspension was used for each well. After $1 \mathrm{hr}$ of incubation $\left(37^{\circ} \mathrm{C} ; 5 \% \mathrm{CO}_{2}\right)$, the glass tiles with the attached cells were transferred into new culture wells. The tiles were washed carefully with culture medium and finally incubated in $1 \mathrm{ml}$ of estrogen-free fresh culture medium supplemented with basic FGF (50 ng/ml; Life Technologies, St. Paul, MN) at $37^{\circ} \mathrm{C}$ in an atmosphere of $5 \% \mathrm{CO}_{2}$. The medium was changed every second day. The purity of the neuronal culture was tested by immunohistochemistry. The following antibodies were used: GFAP (1:100; Linaris, Wertheim-Dettingen, Germany), anti-MAP-2 (1:100; Boehringer, Mannheim, Germany), and anti-myelin/ oligodendrocyte-specific protein (1:1000; Chemicon, Hofheim, Germany). The culture consisted of neurons $(\sim 80 \%)$, astrocytes $(\sim 10 \%)$, and oligodendrocytes $(\sim 10 \%)$. The preparation medium consisted of Hybernate A and B27 (1\%) (both from Life Technologies, Eggenstein, Germany). The estrogen-free culture medium consisted of Neurobasal (without phenol red), B27 (1\%), L-glutamine (500 mM), and antibiotics (1\%) (all from Life Technologies, Eggenstein, Germany). The digestion solution consisted of the preparation medium and papain $(0.2 \% \mathrm{w} / \mathrm{v}$; Sigma).

Treatment of cultures with estradiol or letrozole. After $4 \mathrm{~d}$ in vitro (DIV), the incubation media were supplemented with various doses of $17 \beta$ estradiol $\left(10^{-7}, 10^{-10}\right.$, and $10^{-12} \mathrm{M}$; water soluble; Sigma) or various doses of letrozole $\left(10^{-13}\right.$ to $10^{-7} \mathrm{M}$; kindly provided by Novartis, Basel, Switzerland) for an additional $4 \mathrm{~d}$ (unless mentioned otherwise). Letrozole did not affect the viability of the cultures as tested in pilot experiments (data not shown).

Radioimmunoassay. Medium of nontreated and letrozole-treated cultures was collected every day and pooled for estradiol measurement. The processing of the medium and the measurement itself were performed as described previously (Prange-Kiel et al., 2003). Values measured in unconditioned medium (pure medium, which had not been used for culture) were subtracted as background. For each treatment and each dose, three cultures were measured.

Electron microscopy. After fixation in $1 \%$ glutaraldehyde and $1 \%$ paraformaldehyde in $0.1 \mathrm{~m}$ phosphate buffer ( $\mathrm{PB} ; \mathrm{pH} 7.4$ ), hippocampal slices were postfixed in $1 \% \mathrm{OsO}_{4}$ for $30 \mathrm{~min}$, dehydrated in graded ethanol using $1 \%$ uranyl acetate in $70 \%$ ethanol for $30 \mathrm{~min}$, and embedded in Epon 820 (Serva, Heidelberg, Germany) (Rune et al., 1992). Blocks were trimmed to contain only the stratum pyramidale and radiatum of the CA1 region. Thin sections were cut on a Reichert-Jung OmU3 ultramicrotome. Ultrathin sections were stained with uranyl acetate, followed by lead citrate.

Slice cultures $(n=5)$ from pups of different litters were evaluated for the density of synapses. The spine synapse density was calculated using unbiased stereological methods as described previously (Prange-Kiel et al., 2004). Briefly, pairs of consecutive serial ultrathin sections were cut and collected on formvar-coated single grids. The sections contained the upper and middle third of the CA1 stratum radiatum. Photographs were made at a magnification of $6600 \times$ with the observer blinded to the experimental treatment. Areas occupied by interfering structures, such as large dendrites or blood vessels, were avoided intentionally. To obtain a comparable measure of synaptic numbers, unbiased for possible changes in synaptic size, the disector technique was used (Sterio, 1984). The density of spine synapses of pyramidal cell dendrites was calculated with the help of a reference grid superimposed on the EM prints. Only those spine synapses were counted that were present on the reference section but not on the look-up section. The disector volume was calculated by multiplying the unit area of the reference grid by the distance $(0.09 \mu \mathrm{m})$ between the upper faces of the reference and the look-up section (Braendgaard and Gundersen, 1986). To increase the efficiency of spine synapse counting, the analysis was performed treating each reference section as a look-up section and vice versa (Woolley and McEwen, 1992). At least 10 neuropil fields were photographed on each EM grid. With at least two grids from each slice, containing at least two pairs of consecutive ultrathin sections, each slice provided a minimum of 20 neuropil fields. The mean $\pm \mathrm{SD}$ was calculated. Means were compared using ANOVA, followed by a post hoc test (LSD). A level of confidence of $p<0.05$ was adopted.

Immunolabeling and image analysis. Immunohistochemistry was performed as described previously (Rune et al., 2002). Frozen sections of hippocampal slice cultures or cell cultures were fixed in $4 \%$ paraformaldehyde and incubated overnight at $4^{\circ} \mathrm{C}$ with primary antibodies against synaptophysin (1:1000; Boehringer), ER $\alpha$ (1:10; Santa Cruz Biotechnology, Heidelberg, Germany), ER $\beta$ (1:80; Advanced Biomedical Research, Hackensack, NJ), and spinophilin (1:750; Upstate Biotechnology, Lake Placid, NY). Nuclei were counterstained with TOTO-3 (Upstate Biotechnology) or 4',6-diamidino-2-phenylindole (Sigma).

For the observation and documentation of the samples, a laserscanning microscope (SP2; Leica, Berlin, Germany) was used. The digitized images were further analyzed by a cell imaging system (Openlab 2.2.5; Improvision, Coventry, UK). Deconvolution of the images preceded the analysis, which was performed as described previously (Rune et al., 2002). Briefly, with all measurements, a threshold of signal intensity was defined as being specific. For ER analysis, the nuclear area was deter- 
mined by the TOTO staining. A relative staining index for ER $\alpha$ and ER $\beta$ was calculated by multiplying the stained area (number of pixels) by the intensity of staining (indicated value on a gray scale) and dividing the result by the number of measured cells. One hundred cells each were examined in regions CA1 and CA3 of the slice culture. To assay synaptophysin and spinophilin staining in slice cultures, areas of a defined size were analyzed. Here, a relative staining index was determined by multiplying the stained area (number of pixels) with the intensity of staining (indicated value on a gray scale) and dividing the result by the number of measured areas. For each group ( $n=5$ slices), 20 fields in the stratum radiatum of the CA1 region were analyzed. For synaptophysin and spinophilin measurements in dispersion cultures, the cytoplasm of individual cells including their processes $(n=20)$ was analyzed. In single cells, immunoreactive areas were defined by the threshold of signal intensity and multiplied by the intensity value, resulting in a staining index per cell. Only cells with a clearly discernible nucleus were used. To avoid bias, all analyses were performed with the investigator blind to the protocol of the sample under study. Means \pm SD were calculated. Statistical analyses were performed by ANOVA, followed by a post hoc (Dunnett') test; $p<$ 0.05 was considered to be significant.

Biocytin filling of neurons in slice cultures. CA1 pyramidal neurons in hippocampal slice cultures were intracellularly filled with biocytin using the whole-cell configuration of the patch-clamp technique. For this purpose, whole-cell recordings were made under visual control using differential interference contrast video microscopy. An upright microscope (Axioskop FS; Zeiss, Oberkochem, Germany) equipped with a $40 \times$ immersion objective camera (C2400; Hamamatsu, Hamamatsu City, Japan) was used. Patch pipettes were pulled from borosilicate glass tubes (2.0 mm diameter, $0.5 \mathrm{~mm}$ wall thickness; Hilgenberg, Malzfeld, Germany). When filled with intracellular solution, they had resistances of 3-5 M $\Omega$. Neurons were approached while positive pressure was applied to the inside of the patch pipette. Tight-seal $(>2 \mathrm{G} \Omega)$ whole-cell recordings were established in the pyramidal neurons using an Axopatch 200B amplifier (Axon Instruments, Foster City, CA).

Neurons were filled with biocytin for 10-15 min. After withdrawal of the pipette, cultures were stored for $30 \mathrm{~min}$ in oxygenated medium to allow biocytin transport. Slices were fixed by immersion in a phosphatebuffered solution (0.1 м PB; pH 7.4) containing $1 \%$ paraformaldehyde and $2.5 \%$ glutaraldehyde $\left(12 \mathrm{hr}\right.$ at $\left.4^{\circ} \mathrm{C}\right)$. Cultures were rinsed several times in PB. To block endogenous peroxidase, cultures were transferred to phosphate-buffered $3 \% \mathrm{H}_{2} \mathrm{O}_{2}$ for 10 min, rinsed several times in PBS, and incubated overnight $\left(4^{\circ} \mathrm{C}\right)$ in an ABC-Elite solution (1:100; ALEXIS, Grünberg, Germany), to which $0.1 \%$ Triton X-100 was added. After washing the cultures in PBS, biocytin was visualized using $0.05 \% \mathrm{DAB}$ in PBS containing $0.005 \%(\mathrm{NH} 4)_{2} \mathrm{Ni}(\mathrm{SO} 4)_{2}$ and $0.006 \% \mathrm{COCl}_{2}$ for intensification. Neurons were developed by adding $0.01 \% \mathrm{H}_{2} \mathrm{O}_{2}$ to the DAB solution until all processes of the neurons, including dendritic spines, were clearly visible. Cultures were postfixed in $0.1 \%$ osmium tetroxide in PBS for $\sim 2 \mathrm{~min}$, dehydrated through an ascending series of ethanol ( $50 \%$ to absolute ethanol; $15 \mathrm{~min}$ each step; followed by toluol, $10 \mathrm{~min}$ ), and finally embedded in Hypermount (Lifescience International, Frankfurt, Germany).

Lucifer yellow filling of neurons in dispersion cultures. Cells in dispersion cultures were intracellularly filled with Lucifer yellow ( $5 \%$ in $0.1 \mathrm{M} \mathrm{PB})$, followed by photoconversion of the fluorescent dye into a stable DAB product using a UV lamp $(\lambda=410 \mathrm{~nm})$, as described in detail previously (Naumann et al., 1992a,b).

Morphological analysis of spine density. Light microscopic analysis of neuronal elements and spine counts were performed using a plan APO $100 \times, 1.4$ oil immersion objective attached to a BX50 microscope (Olympus, Hamburg, Germany). Spine counting in cultures containing biocytin- or Lucifer yellow-filled pyramidal cells of each experimental group was performed with the investigator blind to the experimental protocol. Spines were identified as 1 - to $3-\mu \mathrm{m}$-long protrusions from the dendrite, several of them bearing a distinct spine head. For quantification and distribution of spines, camera lucida drawings were performed, and the apical stem dendrite was subdivided into segments of $50 \mu \mathrm{m}$ length (25 $\mu \mathrm{m}$ in dispersion cultures), beginning with the origin of the apical stem dendrite from the soma. In slice cultures, a second set of five seg-

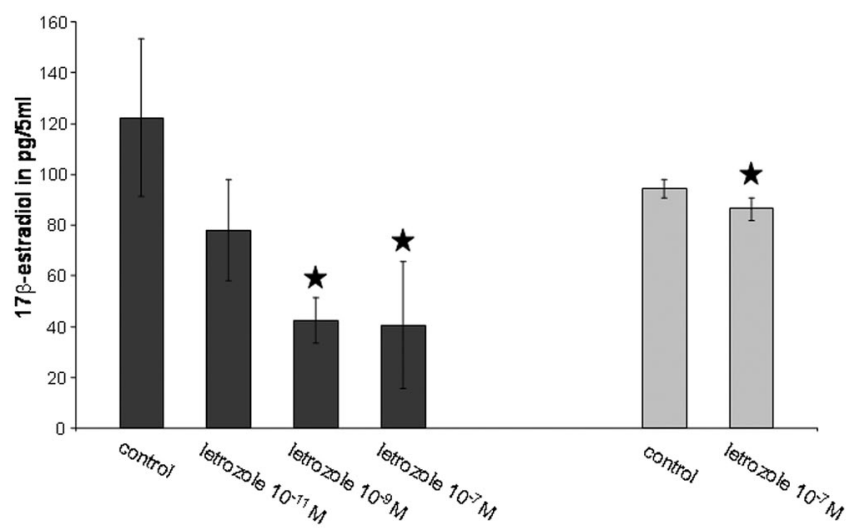

Figure 1. Letrozole inhibits estrogen synthesis. A dose-dependent decrease in $17 \beta$ estradiol release into the medium was found after treatment of slice cultures with various doses of letrozole ( $\square$; mean \pm SD; $n=3$ cultures per group; $\star p<0.05$ to control). Estradiol synthesis was significantly reduced at a dose of $10^{-7} \mathrm{M}$ letrozole in dispersion cultures (鮝; mean $\pm S D ; n=3$ cultures per group; $\star p<0.05$ to control).

ments (50 $\mu \mathrm{m}$ each) of apical third-order dendrites was analyzed. The number of spines in each dendritic segment was counted three times, and means \pm SD were used for statistical analysis. Because the number of spines in each dendritic segment of pyramidal cells showed a normal distribution, the two-way $F$ test was performed. This test was used to reveal a statistically significant difference between variants of the two independent groups of estradiol-treated cultures and untreated controls. The level of significance was set at $p<0.01$.

\section{Results}

\section{Hippocampal estrogen synthesis is regulated by} aromatase activity

In a previous study, we were able to show that adult hippocampal neurons are capable of synthesizing estradiol in vitro (PrangeKiel et al., 2003). In the present study, we used hippocampal slice cultures and neonatal hippocampal dispersion cultures, two established culture systems (Murphy and Segal, 1996; Murphy et al., 1998; Pozzo-Miller et al., 1999), to demonstrate the effects of hippocampus-derived estradiol on spine formation.

In both culture systems, measurement of estradiol in collected media revealed considerable amounts of $17 \beta$-estradiol after 8 DIV (Fig. 1). Supplementation of the medium with letrozole at various doses after 4 DIV led to a significant downregulation of estradiol synthesis. A dose dependency was seen in hippocampal slice cultures at doses in a range of $10^{-11}$ to $10^{-7} \mathrm{M}$ letrozole. At a dose of $10^{-7} \mathrm{M}$, inhibition was maximal (Fig. 1). No similar dose dependency could be achieved in dispersion cultures (data not shown), although at all doses, the mean value was lower than in control cultures. Differences between the various doses, however, were very small and not significant because of a high SD. Probably, the sensitivity of our RIA was too low to discriminate between steps of $3 \mathrm{pm}$ estradiol. With $10^{-7} \mathrm{M}$ letrozole, however, a dose that resulted in maximal inhibition of estradiol synthesis in slice cultures, we also measured a significant downregulation of estradiol synthesis in dispersion cultures (Fig. 1).

\section{Hippocampal synapses are responsive to endogenous estradiol}

Cell viability, as judged by morphological inspection, was not affected by estradiol or letrozole treatment of dispersion cultures. In hippocampal slice cultures, neuronal ultrastructure and the typical cellular arrangement of the pyramidal layer were well preserved and did not differ from the in vivo situation. By electron microscopy, 
no qualitative differences were found between nontreated or estradiol- and letrozole-treated hippocampal slices. In all groups of cultures, spine synapses could be distinguished from shaft synapses in the CA1 region (Fig. 2A-F), where estrogen-induced spine formation had been described in previous studies.

As a first step in our quantitative EM analysis, we determined the number of presynaptic boutons (identified by the presence of synaptic vesicles), the number of spine synapses, and the number of shaft synapses in stratum radiatum (CA1) of slice cultures using unbiased stereological methods. At a dose of estradiol used in the study by Pozzo-Miller et al. (1999), who noticed an increase in spine number by $100 \%$ after estradiol treatment of hippocampal slice cultures, we were unable to find an effect of estradiol on the number of spine and shaft synapses and of boutons (Fig. 2G). However, our cultures were responsive to estrogen. Application of estradiol at a dose of $10^{-7} \mathrm{M}$ reliably led to an upregulation of ERs (see below). In contrast, inhibition of estradiol synthesis by letrozole resulted in a significant downregulation of spine synapses and of presynaptic boutons (Fig. 2G). No changes were found in the number of shaft synapses after incubation with letrozole.

Our EM findings were confirmed by spine counts in Lucifer yellow-filled neurons in dispersion cultures (Fig. 3). In accordance with previous studies (Amateau and McCarthy, 2002), spine density was generally much lower in hippocampal dispersion cultures compared with organotypic hippocampal cultures, likely because of a decreased number of synaptic connections in these dissociated neurons. Estradiol treatment did not significantly increase the number of spines, whereas a significant reduction in spine number was seen after incubation with letrozole at a dose of $10^{-7} \mathrm{M}$ (Fig. 3).

Next, we also determined the number of spines on apical dendrites of biocytin-

filled CA1 pyramidal cells in hippocampal slice cultures. These studies were performed in view of previous reports indicating an increased spine density in slice cultures treated with estradiol (Pozzo-Miller et al., 1999). According to Pozzo-Miller et al. (1999), our cultures were treated with estradiol at a dose of $7 \times$ $10^{-7}$ M. To exclude gender differences, slice cultures of male $(n=$ $8)$ and female $(n=8)$ animals were used. As shown in Figure $4 A$, biocytin-injected cells in these cultures showed a similar dendritic arborization as known from Golgi-impregnated pyramidal cells in perfusion-fixed hippocampi. Dendritic spines were clearly discernible (inset). Also, the distribution pattern of spines along the apical stem dendrite of nontreated CA1 neurons was in line with previous reports (Table 1 ). We found $\sim 25 \%$ more spines than in the controls in the study by Pozzo-Miller et al.
(1999). Statistical analysis using the two-way $F$ test did not reveal significant differences in spine density along apical stem dendrites of CA1 pyramidal cells cultured in the presence or absence of exogenous estradiol (Fig. $4 B$ ). Because significant differences in spine number were found on tertiary dendrites after estradiol application (Pozzo-Miller et al., 1999), we additionally counted spines on tertiary dendrites of CA1 pyramidal cells (Fig. 4C). In accordance with the spine counts on apical dendrites, estradiol treatment did not result in a significant change in spine number on tertiary dendritic branches. No differences were found between slices from male and female animals.

Together, our fine structural studies as well as the spine counts in hippocampal cell cultures and slice cultures do not support previous reports on the effects of exogenously applied estrogen 


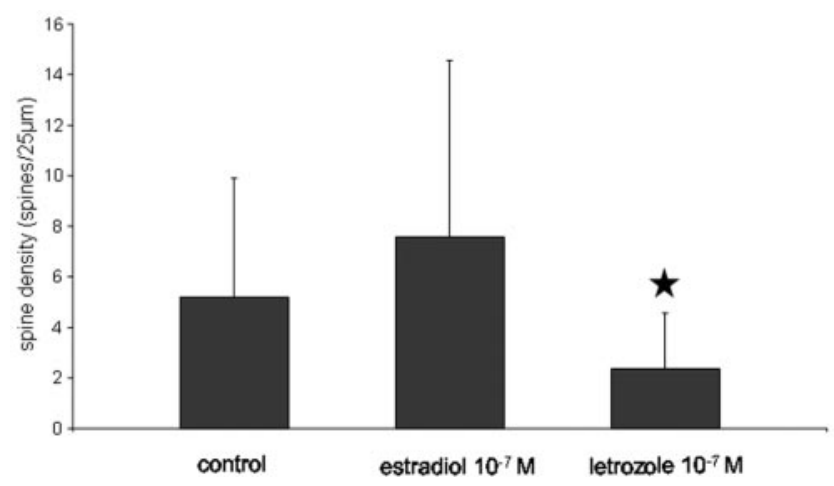

Figure 3. Letrozole reduces spine density in cultured hippocampal neurons. In hippocampal dispersion cultures, letrozole significantly reduces spine density on apical dendrites of Lucifer yellow-stained and photoconverted pyramidal neurons ( $n=16$ cells per group; mean $\pm S D$; $\star p<0.01$ to control). No significant effect was seen after estradiol treatment.

on spinogenesis. However, they point to a role of local estrogen synthesis in the maintenance of spines because both spines and spine synapses were reduced after incubation with the aromatase inhibitor letrozole. In other words, reducing estrogen synthesis may be a more sensitive approach to study estrogen effects on synapse structures than exogenous application of the hormone.

\section{Aromatase inhibition downregulates synaptic protein expression}

Next, we asked for the regulation of synaptic proteins in response to local estrogen synthesis. We chose synaptophysin as a presynaptic marker and spinophilin as a marker of postsynaptic spines.

Spinophilin, a protein highly enriched in dendritic spines, has been implicated in regulating spine formation and function (Feng et al., 2000; Hao et al., 2003; Muly et al., 2004). We therefore used this protein as a marker of spines, speculating that an increase in spinophilin expression might reflect an increase in spine number (Amateau and McCarthy, 2002). In hippocampal slice cultures and dispersion cultures, immunoreactivity was found in the cell soma and in dendrites (Fig. 5A-D) but appeared also as punctate staining, indicating the labeling of spines (Fig. $5 D)$. After treatment with letrozole, staining intensity as revealed by image analysis was dramatically reduced (Fig. $5 E$ ). Consonant changes induced by letrozole were observed in dispersion cultures and quantified by image analysis (Fig. $5 F$ ). An upregulation of spinophilin in response to estradiol, which we expected, was not found by quantitative immunohistochemistry, neither in the CA1 region of slice cultures (Fig. $5 E$ ) nor in dispersion cultures (Fig. $5 F)$.

Synaptophysin, being a constituent of the synaptic vesicle membrane, is a well established presynaptic marker. It has been shown to be responsive to estradiol application (Rune et al., 2002; Yokomaku et al., 2003). In slice cultures and in dispersion cultures, immunoreactivity appeared as a punctate staining (Fig. $6 A-D)$. After letrozole treatment, a clear-cut downregulation of synaptophysin expression was found by image analysis of immunolabeling in both types of cultures (Fig. 6E,F). After treatment with estradiol, there was an upregulation of synaptophysin in slice cultures as well as in dispersion cultures (Fig. 6E,F), thus confirming previous findings by Yokomaku et al. (2003). Upregulation of synaptophysin not accompanied by an increase in bouton number as found by counting boutons in electron micrographs (Fig. 2G) points to a direct effect of estradiol on this protein and confirms the study by Yokomaku et al. (2003).

\section{Control experiments}

Ligand-induced ER expression, which we routinely performed to control for the responsiveness of the cultures to estrogen, was found similarly regulated in the present experiments as described previously (Rune et al., 2002; Prange-Kiel et al., 2003). In dispersion cultures, $\mathrm{ER} \alpha$ was upregulated, whereas $\mathrm{ER} \beta$ expression was decreased in response to estradiol. In slice cultures, an upregulation was found with $\mathrm{ER} \alpha$, particularly in CA1. $\mathrm{ER} \beta$ expression was not changed in slice cultures by treatment with estradiol (data not shown).

In a "rescue" experiment, cells in dispersion cultures were treated with letrozole or estradiol after 4 DIV, and then synaptophysin expression was analyzed $24 \mathrm{hr}$ later. As mentioned before, letrozole treatment resulted in a significant decrease of synaptophysin expression. We found that synaptophysin expression could be restored by adding estradiol together with letrozole to the medium at a dose of $10^{-7} \mathrm{M}$ (Fig. 7). Finally, we wanted to test the effects of estradiol plasma concentrations $\left(10^{-12} \mathrm{M}\right.$ in male and $10^{-10} \mathrm{M}$ in female rats) in cultures that had not been treated with letrozole before. Under these conditions, no upregulation of synaptophysin in response to estradiol was found, neither at a dose of $10^{-12} \mathrm{M}$ in hippocampal dispersion cultures from male rats nor at a dose of $10^{-10} \mathrm{M}$ in cultures from female rats (Fig. 8). The downregulation of synaptophysin by letrozole could not be restored when the treatment was accompanied by estradiol application at normal plasma concentrations of male and female rats (male, $10^{-12} \mathrm{M}$; female, $10^{-10} \mathrm{M}$ ) to cultures from male and female animals, respectively (Fig. 8).

\section{Discussion}

The results of the present study show, for the first time, that inhibition of aromatase activity in hippocampal cultures and the subsequent downregulation of local estrogen synthesis decreases the number of spine synapses in hippocampal cultures. Interestingly enough, opposite effects were not observed by treatment of the cultures with estradiol. Our findings point to an essential role of hippocampus-derived estrogen in the maintenance of hippocampal synapses.

\section{Letrozole as a tool to study estrogen effects in neural tissue}

The concept that steroids are synthesized de novo in the brain has emerged from observations made in the 1980s by Baulieu and coworkers (for review, see Baulieu, 1997). Results from several laboratories over the past decade have established unequivocally that the enzymes found in classic steroidogenic tissues are present in the CNS (Compagnone and Mellon, 2000). Aromatase, which transforms testosterone to estradiol, has been detected in rat hippocampal neurons both at the mRNA level (Abdelgadir et al., 1994; Wehrenberg et al., 2001) and protein level (Sanghera et al., 1991; Garcia-Segura et al., 1999, 2001). Recently, it was shown that adult hippocampal neurons indeed synthesize estrogens (Prange-Kiel et al., 2003). The conversion of androgens to estrogens by aromatase is the most crucial step in the estrogen synthesis pathway. Irreversible steroidal agents inactivate the enzyme, whereas reversible nonsteroidal inhibitors, such as letrozole, actively decrease the amount of circulating estrogens (Foidart et al., 1995; Geisler et al., 2002). Letrozole turned out to be the most potent aromatase inhibitor in many culture systems. In contrast to most antiestrogens (for review, see Nilsson and Gustafsson, 2002) and other inhibitors of steroidogenesis, it does not interfere with ER function (Puddefoot et al., 2002; Prange-Kiel et al., 2003). In adult hippocampal dispersion cultures, estrogen synthesis could be almost completely inhibited by letrozole at a dose 
of $10^{-9} \mathrm{M}$ (Prange-Kiel et al., 2003). In the present study, estradiol withdrawal by letrozole treatment resulted in a downregulation of spinophilin and synaptophysin expression. The latter effect could be restored by simultaneous application of estradiol and letrozole. This finding underscores the specific effect of letrozole on aromatase and makes possible toxic effects of doses used in this study unlikely. With both parameters, synthesis of estrogen and synaptophysin, maximal effects were found at a dose of $10^{-7} \mathrm{M}$ letrozole, thus confirming previous studies on letrozole effects in other culture systems (Mitropoulou et al., 2003).

\section{A role of hippocampal aromatase for the maintenance of synapses}

Our data show that reducing estradiol levels by blocking aromatase activity with letrozole reduces dendritic spine synapse density and downregulates postsynaptic proteins such as spinophilin. Presynaptically, synaptophysin expression was downregulated, and a loss of boutons was found. This points to a crucial role of brain aromatase and local estrogen synthesis in the maintenance of spine synapses. A key role for aromatase has been described frequently in studies on neuroprotective effects of estrogen (for review, see Azcoitia et al., 2001). For instance, intracerebral administration of fadrozole, another aromatase inhibitor, enhanced kainic acid-induced neurodegeneration in the hippocampus of male rats (Peterson et al., 2001). Aromatase is upregulated in the adult nervous system in different lesion paradigms (Garcia-Segura et al., 1999), as are ERs after brain injury (Dubal et al., 1999). This suggests that the endogenous response of neural tissue to cope with cerebral insults may include the induction of aromatase and the consecutive increase in local estradiol synthesis. Estradiol, in turn, upregulates ERs, particularly in CA1, where an estradiol-induced spine increase was found in previous studies. For hippocampal neurons, this dependency of ER expression on hippocampal estrogen synthesis was recently shown (Prange-Kiel et al., 2003). Because the sensitivity of hippocampal neurons in our system is higher to lowering estrogen levels by aromatase inhibition than to application of estradiol, our findings confirm the concept of aromatase and local estrogen synthesis as being strong neuroprotective factors in the hippocampus, in this case by maintaining hippocampal spine synapses.

\section{Does estrogen induce synapse formation?}

Our findings contrast to the widely held concept of estrogeninduced spine formation in vivo (Gould et al., 1990; Woolley et al., 1990; Woolley and McEwen, 1993; Segal and Murphy, 2001; McEwen, 2002) and in vitro (Murphy and Segal, 1996, 1998; Pozzo-Miller et al., 1999) because not the application of estradiol, but lowering estrogen levels, was found to be effective.

The conflicting results between previous in vitro studies and our in vitro findings may indicate a ceiling under the current conditions that impedes additional spine increase. However, under the experimental conditions of previous reports, the maximal spine number may not have been reached, allowing an additional increase in spine density. Pozzo-Miller et al. (1999) found a clear-
Table 1. Spine distribution on apical dendrites of biocytin-filled CA1 pyramidal neurons in control slice cultures

\begin{tabular}{ll}
\hline Apical stem dendrite segment $(\mu \mathrm{m})$ & Spine density $($ spines $/ \mu \mathrm{m})$ \\
\hline $0-50$ & $0.87 \pm 0.17$ \\
$50-100$ & $1.54 \pm 0.15$ \\
$100-150$ & $1.89 \pm 0.23$ \\
$150-200$ & $1.87 \pm 0.18$ \\
$200-250$ & $1.60 \pm 0.17$ \\
\hline
\end{tabular}

After $8 \mathrm{~d}$ in culture, CA1 pyramidal cells in control slice cultures displayed a distribution of spines along the apical stem dendrite similar to that published in previous reports $(n=16$; mean \pm SD).

cut upregulation of spines in response to estradiol in slice cultures and Murphy and Segal (1996) in dispersion cultures. The differences may be attributable to the age of the animals used [for instance, Murphy and Segal (1996) used cultures of prenatal instead of postnatal animals] as well as to other factors that may interact with estradiol. In both studies, serum was added to the culture medium, which not only contained various undefined steroids but also growth factors. Several growth factors, in turn, were shown to be regulated by estradiol (Toran-Allerand, 2000). Previously, estrogen-induced upregulation of spines has not been shown under serum-free and steroid-free culture conditions.

Several in vivo studies have already shown that exogenous estradiol does not directly increase hippocampal spine density. It has been known for at least 10 years that changes in spine density during the estrous cycle do involve more than estradiol alone. Gould et al. (1990), using adult female animals, were the first to show estradiol-induced changes in spine density but also indicated a role for progesterone in regulating spine density. Woolley et al. (1990) showed varying spine densities during the estrous cycle, and Woolley and McEwen (1993) also demonstrated a critical role for progesterone in producing a decrease in spine density between proestrus and estrus stages of the cycle. This decrease could be blocked by an antiprogestin. Furthermore, progesterone downregulates ERs which are widely distributed in the hip- 

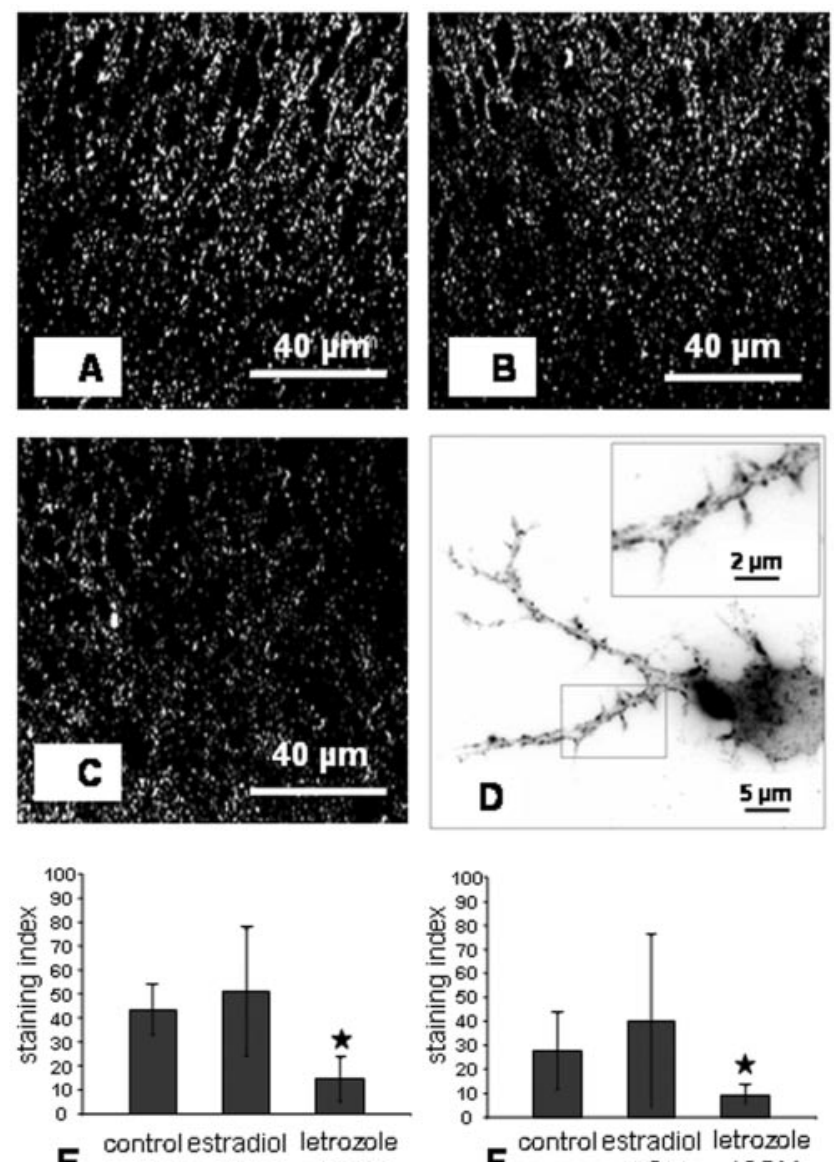

E $10^{-7} \mathrm{M} \quad 10^{-7} \mathrm{M}$

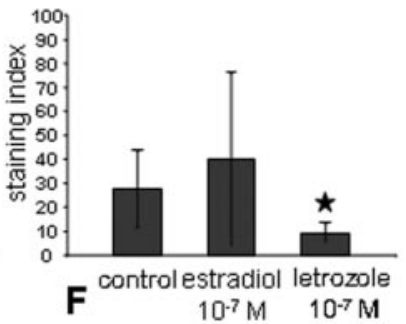

Figure 5. Immunostaining for spinophilin. $A-C$, Stratum radiatum of CA1 in hippocampal slice cultures. Immunoreactivity for spinophilin in an estradiol-treated slice culture $(A)$, control slice culture $(B)$, and letrozole-treated slice culture ( $C$. D, Punctate spinophilin immunoreactivity on dendrites of a single pyramidal cell in dispersion culture (control). $E$, Image analysis of spinophilin immunolabeling in the stratum radiatum of CA1 after estradiol or letrozole treatment. Letrozole treatment resulted in a significant downregulation of spinophilin. An upregulation by estradiol was not found ( $n=5$ slices per group; mean \pm SD; $\star p<0.05$ ). $F$, Image analysis of spinophilin immunolabeling in single pyramidal cells after estradiol and letrozole treatment, respectively. Letrozole led to a significant downregulation of spinophilin immunoreactivity ( $n=20$ cells per group; mean $\pm \mathrm{SD} ; \star p<0.05$ to control).

pocampus (Azcoitia et al., 1999; Hösli and Hösli, 1999; Gundlah et al., 2000; Hart et al., 2001; Shughrue and Merchenthaler, 2001; Wehrenberg et al., 2001; Rune et al., 2002).

Along this line, treatment of ovariectomized rats with estradiol, which results in an increase in spines on apical dendrites of CA1 pyramidal cells (for review, see Segal and Murphy, 2001; McEwen, 2002), does not prove direct effects of exogenously applied estradiol on spine or synapse formation. The physiological balance of estradiol interaction with other factors (e.g., neurotrophins) is far from being understood (Toran-Allerand, 2000), and physiological concentrations of estradiol in various brain regions are yet unknown. In addition, it has become obvious during the last decade that estrogen synthesis takes place in many tissues. For instance, considerable amounts of estradiol and other steroid metabolites are synthesized in bone, fat tissue, and in the adrenal glands (Simpson et al., 1999). Exogenous estrogen application to animals is likely to interfere with a fine-regulated endogenous balance. Furthermore, there is increasing evidence that afferent connections of the hippocampus that arise from estrogensensitive subcortical regions play a role in estrogen-induced hip-
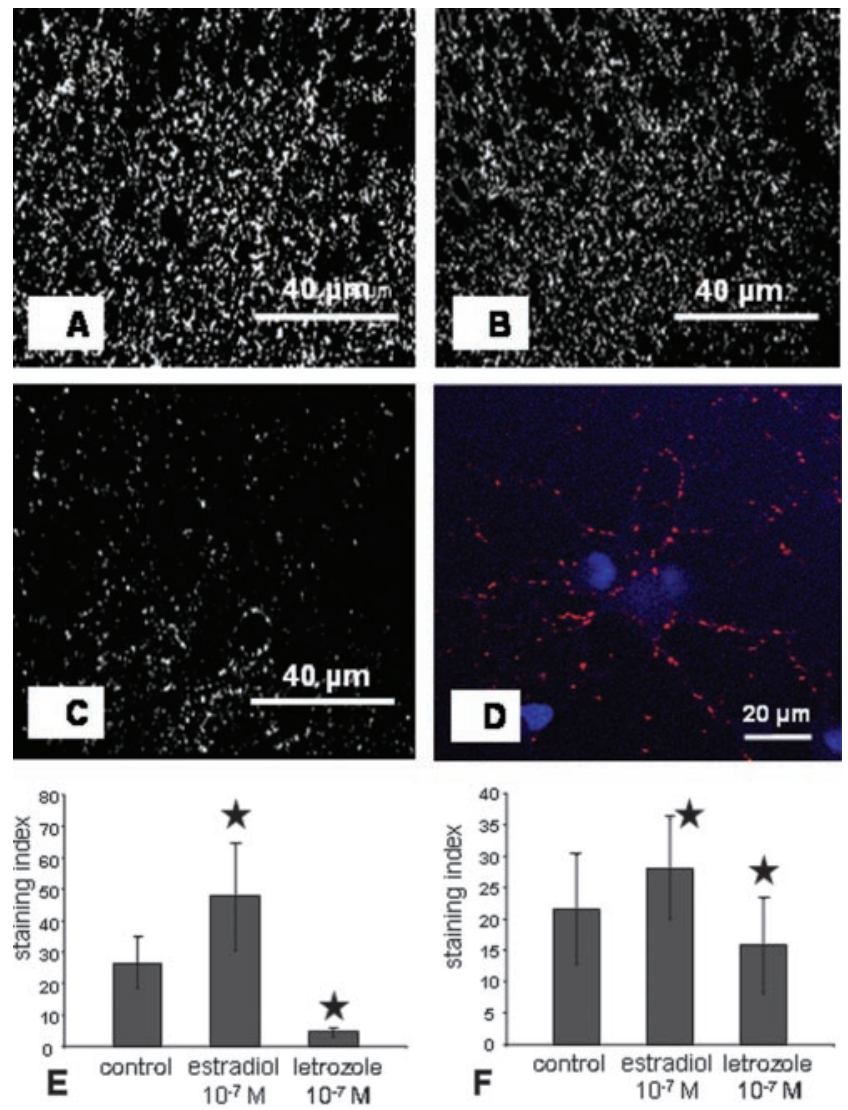

Figure 6. Immunoreactivity for synaptophysin. A-C, Stratum radiatum of CA1 in hippocampal slice cultures. Immunoreactivity of synaptophysin in estradiol-treated slice culture $(A)$, control slice culture $(B)$, and letrozole-treated slice culture ( $C$. D, Synaptophysin immunoreactivity in a dispersion culture. Red puncta outline dendrites. Cell nuclei are counterstained with DAPI (blue). $E$, Image analysis of synaptophysin immunostaining in the stratum radiatum of CA1. Estradiol significantly upregulates and letrozole downregulates immunostaining for synaptophysin ( $n=5$ slices per group; mean \pm SD; $\star p<0.05$ to control). $F$, Image analysis of synaptophysin in single pyramidal cells after estradiol and letrozole treatment, respectively. Letrozole treatment resulted in a significant downregulation of synaptophysin immunoreactivity. Conversely, intensity of immunolabeling was increased after treatment with estradiol ( $n=$ 20 cells per group; mean \pm SD; $\star p<0.05$ ).

pocampal plasticity (Leranth et al., 2000; Lam and Leranth, 2003; Prange-Kiel et al., 2004). Estrogen replacement in ovariectomized rats had no effect on spine density in the hippocampus after fimbria/fornix lesion, which suggests that hormonal regulation of hippocampal spine synapse density involves subcortical control (Leranth et al., 2000). Recently, Rudnick et al. (2003) showed that estrogen-regulated synaptic plasticity partly depends on basal forebrain cholinergic neurons.

In the present study, synaptophysin expression was upregulated by estrogens, but this was not accompanied by a change in bouton number. These findings confirm our previous results (Rune et al., 2002) as well as findings by Yankova et al. (2001) in vivo and Yokomaku et al. (2003) in vitro. They found an increase in multi-synapse boutons in response to estradiol but not more boutons (Yankova et al., 2001). Yokomaku et al. (2003) also showed that estrogen-induced upregulation of synaptophysin was not accompanied by changes in the number of immunoreactive boutons. Thus, estradiol may directly activate the exocytotic machinery as shown by an upregulation of presynaptic proteins and enhanced glutamate release (Yokomaku et al. 2003). There was no estrogen-induced upregulation of spinophilin in the present exper- 


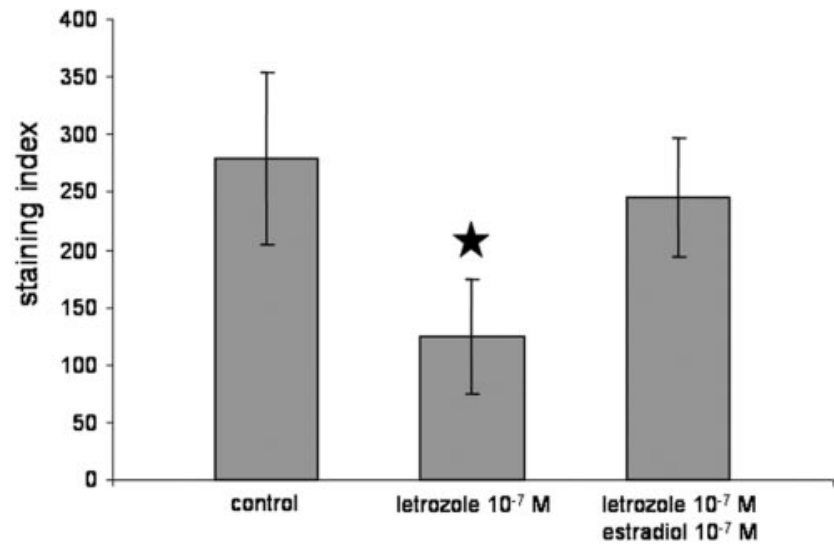

Figure 7. "Rescue" of synaptophysin labeling after treatment with letrozole. After $24 \mathrm{hr}$ of treatment with $10^{-7} \mathrm{M}$ letrozole, a significant downregulation of synaptophysin immunolabeling was found. This effect could be restored if the medium was supplemented with estradiol, together with letrozole ( $n=20$; mean \pm SD; $\star p<0.05$ to control).

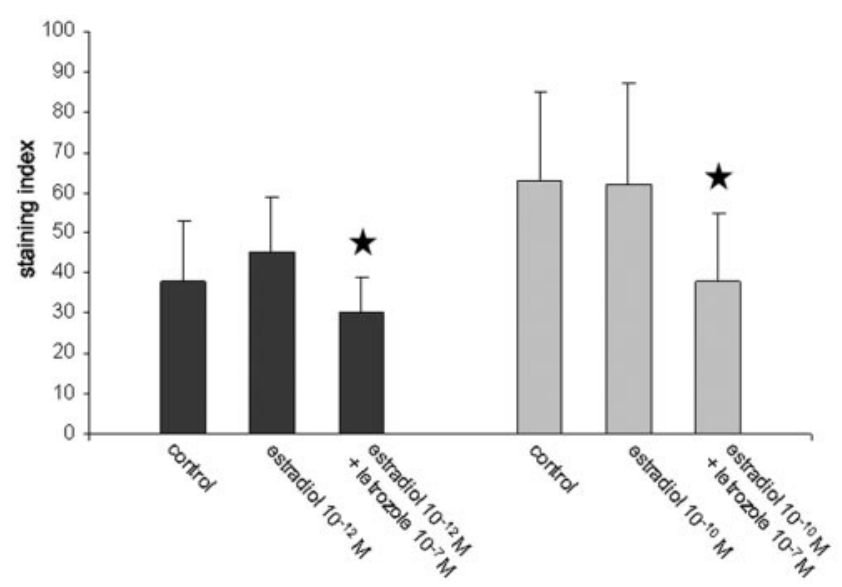

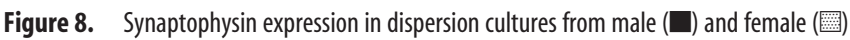
animals. The physiological doses of $10^{-12} \mathrm{~m}$ estradiol for male and $10^{-10} \mathrm{~m}$ estradiol for female animals had no effect on immunolabeling, quantified by image analysis, and the significant downregulation of synaptophysin by letrozole could not be restored by these physiological doses ( $n=20$ cells per group; mean \pm SD; $\star p<0.05$ )

iments, corroborating our morphological findings that no more spines were formed. However, lowering estradiol levels by letrozole caused both downregulation of synaptophysin and spinophilin and also resulted in a decrease in the number of boutons and spines.

In summary, our findings point to an essential role of hippocampus-derived estrogen in the maintenance of hippocampal synapses. The data are consistent with recent studies by Kawato and coworkers (Hojo et al., 2004), who showed that the concentration of estradiol in male hippocampal slices is six times higher than the average levels of estradiol in plasma. They propose brain neurosteroids, including estradiol, to be modulators of interneuronal communication by acting in a paracrine manner. In analogy, it appears from our findings that an intrinsic, fine-regulated estrogen synthesis regulates spine synapses in the hippocampus.

\section{References}

Abdelgadir ES, Resko JA, Ojeda SR, Lephart ED, McPhaul MJ, Roselli CE (1994) Androgens regulate aromatase cytochrome P450 messenger ribonucleic acid in rat brain. Endocrinology 135:395-401.

Akama KT, McEwen BS (2003) Estrogen stimulates postsynaptic density-95 rapid protein synthesis via Akt/protein kinase B pathway. J Neurosci 23:2333-2339.
Amateau SK, McCarthy MM (2002) A novel mechanism of dendritic spine plasticity involving estradiol induction of prostaglandin- $\mathrm{E}_{2}$. J Neurosci 2:8586-8596.

Azcoitia I, Sierra A, Garcia-Segura LM (1999) Localization of estrogen receptor $\beta$-immunoreactivity in astrocytes of the adult rat brain. Glia $26: 260-267$.

Azcoitia I, Sierra A, Veiga S, Honda S, Harada N, Garcia-Segura LM (2001) Brain aromatase is neuroprotective. J Neurobiol 47:318-329.

Balthazart J, Baillien M, Ball GF (2001) Phosphorylation processes mediate rapid changes of brain aromatase activity. J Steroid Biochem 79:261-277.

Baulieu EE (1997) Neurosteroids: of the nervous system, by the nervous system, for the nervous system. Recent Prog Horm Res 52:1-31.

Bi R, Foy MR, Vouimba RM, Thompson RF, Baudry M (2001) Cyclic changes in estradiol regulate synaptic plasticity through the MAP kinase pathway. Proc Natl Acad Sci USA 98:13391-13395.

Björnström L, Sjöberg M (2002) Signal transducers and activators of transcription as downstream targets of nongenomic estrogen receptor actions. Mol Endocrinol 16:2202-2214.

Braendgaard H, Gundersen HJG (1986) The impact of recent stereological advances on quantitative studies of the nervous system. J Neurosci Methods 18:39-78.

Brake WG, Alves SE, Dunlop JC, Lee SJ, Bulloch K, Allen PB, Greengard P, McEwen BS (2001) Novel target sites for estrogen action in dorsal hippocampus: an examination of synaptic proteins. Endocrinology 142:1284-1289.

Brewer GJ (1997) Isolation and culture of rat hippocampal neurons. J Neurosci Methods 71:143-155.

Compagnone NA, Mellon SH (2000) Neurosteroids: biosynthesis and function of these novel neuromodulators. Front Neuroendocrinol 21:1-56.

Crispino M, Stone DJ, Wei M, Anderson CP, Tocco G, Finch CE, Baudry M (1999) Variations of synaptotagmin I, synaptotagmin IV, and synaptophysin mRNA levels in rat hippocampus during the estrous cycle. Exp Neurol 159:574-583.

Dubal DB, Shughrue PJ, Wilson ME, Merchenthaler I, Wise PM (1999) Estradiol modulates bcl-2 in cerebral ischemia: a potential role for estrogen receptors. J Neurosci 19:6385-6393.

Feng J, Yan Z, Ferreira A, Tomozawa K, Liauw, Zhuo M, Allen PB, Quimet CC, Greengard P (2000) Spinophilin regulates the formation and function of dendritic spines. Proc Natl Acad Sci USA 97:9287-9296.

Foidart A, Tlemcani O, Harada N, Abe-Dohmae S, Balthazart J (1995) Preand post-translational regulation of aromatase by steroidal and nonsteroidal aromatase inhibitors. Brain Res 701:267-278.

Frotscher M, Zafirow S, Heimrich B (1995) Development of identified neuronal types and of specific synaptic connections in slice cultures of rat hippocampus. Prog Neurobiol 352:55-68.

Garcia-Segura LM, Wozniak A, Azcoitia I, Rodriguez JR, Hutchinson RE, Hutchinson JB (1999) Aromatase expression by astrocytes after brain injury: implications for local estrogen formation in brain repair. Neuroscience 89:567-578.

Garcia-Segura LM, Azcoitia I, DonCarlos LL (2001) Neuroprotection by estradiol. Prog Neurobiol 63:29-60.

Geisler J, Haynes B, Anker G, Dowsett M, Lonning PE (2002) Influence of letrozole and anastrozole on total body aromatization and plasma estrogen levels in postmenopausal breast cancer patients evaluated in a randomized, cross-over study. J Clin Oncol 20:751-757.

Gould E, Woolley CS, Frankfurt M, McEwen BS (1990) Gonadal steroids regulate dendritic spine density in hippocampal pyramidal cells in adulthood. J Neurosci 10:1286-1291.

Gundlah C, Kohama SG, Mirkes SJ, Garyfollou VT, Urbanski HF, Bethea CL (2000) Distribution of estrogen receptor beta (ER $\beta$ ) mRNA in hypothalamus, midbrain and temporal lobe of spayed macaque: continued expression with hormone replacement. Mol Brain Res 76:191-204.

Hao J, Janssen WG, Tang Y, Roberts JA, McKay H, Lasley B, Allen BP, Greengard P, Rapp PR, Kordower JH, Hof PR, Morrison JH (2003) Estrogen increases the number of spinophilin-immunoreactive spines in the hippocampus of young and aged female rhesus monkeys. J Comp Neurol 465:540-550.

Hart SA, Patton JD, Woolley CS (2001) Quantitative analysis of ER $\alpha$ and GAD colocalization in the hippocampus of the adult female rat. J Comp Neurol 440:144-155.

Hojo Y, Hattori TA, Enami T, Furukawa A, Suzuki K, Ishii HT, Mukai H, Morrison JH, Janssen WG, Kominami S, Harada N, Kimoto T, Kawato S 
(2004) Adult male rat hippocampus synthesizes estradiol from pregnenolone by cytochromes P45017 $\alpha$ and P450 aromatase localized in neurons. Proc Natl Acad Sci USA 101:865-870.

Hösli E, Hösli L (1999) Cellular localization of estrogen receptors on neurons in various regions of cultured rat CNS: coexistence with cholinergic and galanin receptors. Int J Dev Neurosci 4:317-330.

Ing NH, Ott TL (1999) Estradiol up-regulates estrogen receptor- $\alpha$ messenger ribonucleic acid in sheep endometrium by increasing its stability. Biol Reprod 60:134-139.

Ing NH, Spencer TE, Bazer FW (1996) Estrogen enhances endometrial receptor gene expression by a posstranscriptional mechanism in the ovarectomized ewe. Biol Reprod 54:591-599.

Klinge CM (2001) Estrogen receptor interaction with estrogen response elements. Nucleic Acids Res 29:2905-2919.

Lam TT, Leranth C (2003) Role of the medial septum diagonal band of Broca cholinergic neurons in oestrogen-induced spine synapse formation on hippocampal CA1 pyramidal cells of female rats. Eur J Neurosci 17:1997-2005.

Leranth C, Shanabrough M, Horvath TL (2000) Hormonal regulation of hippocampal spine synapse density involves subcortical mediation. Neuroscience 101:349-356.

L'Horset F, Perret C, Brehier A, Thomasset M (1990) 17 $\beta$-Estradiol stimulates the calbindin-D9k (CaBP9k) gene expression at the transcriptional and posttranscriptional levels in the rat uterus. Endocrinology 127:2891-2897.

Linford N, Wade C, Dorsa D (2000) The rapid effects of estrogen are implicated in estrogen-mediated neuroprotection. J Neurocytol 29:367-374.

McEwen BS (2002) Estrogen action throughout the brain. Recent Prog Horm Res 57:357-384.

Mitropoulou TN, Tzanakakis GN, Kletsas D, Kalofonos HP, Karamanos NK (2003) Letrozole as a potent inhibitor of cell proliferation and expression of metalloproteinases (MMP-2 and MMP-9) by human epithelial breast cancer cells. Int J Cancer 104:155-160.

Moss RL, Gu Q, Wong M (1997) Estrogen: nontranscriptional signalling pathway. Recent Prog Horm Res 52:33-67.

Muly EC, Smith Y, Allen P, Greengard P (2004) Subcellular distribution of spinophilin immunolabeling in primate prefrontal cortex: localization to and within dendritic spines. J Com Neurol 469:185-197.

Murphy DD, Segal M (1996) Regulation of dendritic spine density in cultured rat hippocampal neurons by steroid hormones. J Neurosci 16:4059-4068.

Murphy DD, Cole NB, Greenberger V, Segal M (1998) Estradiol increases dendritic spine density by reducing GABA neurotransmission in hippocampal neurons. J Neurosci 18:2550-2559.

Naumann T, Linke R, Frotscher M (1992a) Fine structure of rat septohippocampal neurons: I. Identification of septohippocampal projection neurons by retrograde tracing combined with electron microscopic immunocytochemistry and intracellular staining. J Comp Neurol 325:207-218.

Naumann T, Peterson GM, Frotscher M (1992b) Fine structure of rat septohippocampal neurons: II. A time course analysis following axotomy. J Comp Neurol 325:219-242.

Nilsson S, Gustafsson JA (2002) Estrogen receptor action. Crit Rev Eukaryot Gene Expr 14:237-257.

Peterson RS, Saldanha CJ, Schlinger BA (2001) Rapid upregulation of aromatase mRNA and protein following neuronal injury in the zebra finch (Taeniopygia guttata). J Neuroendocrinol 13:317-323.

Pozzo-Miller LD, Inoue T, Murphy DD (1999) Estradiol increases spine density and NMDA-dependent $\mathrm{CA}^{2+}$ transients in spines of CA1 pyramidal neurons from hippocampal slices. J Neurophysiol 81:1404-1411.

Prange-Kiel J, Wehrenberg U, Jarry H, Rune GM (2003) Para/autocrine regulation of estrogen receptors in hippocampal neurons. Hippocampus 13:226-234.

Prange-Kiel J, Rune GM, Leranth C (2004) Median raphe mediates estrogenic effects to the hippocampus in female rats. Eur J Neurosci 19:309-317.

Puddefoot JR, Barker S, Glover HR, Malouitre SDM, Vinson GP (2002) Non-competitive steroid inhibition of estrogen receptor functions. Int J Cancer 101:17-22.

Razandi M, Pedram A, Greene GL, Levin ER (1999) Cell membrane and nuclear oestrogen receptors (ERs) originate from a single transcript: studies of ER $\alpha$ and ER $\beta$ expressed in Chinese hamster ovary cells. Mol Endocrinol 13:307-319.
Revelli A, Massobrio M, Tessarik J (1998) Nongenomic actions of steroid hormones in reproductive tissues. Endocr Rev 19:3-17.

Rudnick CN, Gibbs RB, Woolley CS (2003) A role for the basal forebrain cholinergic system in estrogen-induced disinhibition of hippocampal pyramidal cells. J Neurosci 23:4479-4490.

Rune GM, Leuchtenberg U, Schröter-Kermani C, Merker HJ (1992) Zonal differentiation of the marmoset (Callthrix jacchus) endometrium. J Anat 181:301-312.

Rune GM, Wehrenberg U, Prange-Kiel J, Zhou L, Adelmann G, Frotscher M (2002) Estrogen up-regulates estrogen receptor alpha and synaptophysin in slice cultures of rat hippocampus. Neuroscience 113:167-175.

Sanghera MK, Simpson ER, McPhaul MJ, Kozlowski G, Conley AJ, Lephart ED (1991) Immunocytochemical distribution of aromatase cytochrome P450 in the rat brain using peptide-generated polyclonal antibodies. Endocrinology 129:2834-2844.

Segal M, Murphy DD (2001) Estradiol induces formation of dendritic spines in hippocampal neurons: functional correlates. Horm Behav 40:156-159.

Shingo AS, Kito S (2002) Estrogen induces elevation of cAMP-dependent protein kinase activity in immortalized hippocampal neurons: Imaging in living cells. J Neural Transm 109:171-174.

Shughrue PJ, Merchenthaler I (2001) Distribution of estrogen receptor $\beta$ immunoreactivity in the rat central nervous system. J Comp Neurol 436:64-81.

Simpson E, Rubin G, Clyne C, Robertson K, O’Donnell L, Davis S, Jones M (1999) Local estrogen biosynthesis in males and females. Endocr Relat Cancer 6:131-137.

Srivastava RAK, Srivastava N, Averna M, Lin RC, Korach KS, Lubahn DB, Schonfeld G (1997) Estrogen up-regulates apolipoprotein E (ApoE) gene expression by increasing ApoE mRNA in the translating pool via the estrogen receptor $\alpha$-mediated pathway. J Biol Chem 272:33360-33366.

Sterio DC (1984) The unbiased estimation of number and sizes of arbitrary particles using the disector. J Microsc 134:127-136.

Stoppini L, Buchs PA, Muller D (1991) A simple method for organotypic cultures of nervous tissue. J Neurosci Methods 37:173-182.

Toran-Allerand CD (2000) Novel sites and mechanisms of oestrogen action in the brain. Novartis Found Symp 230:56-69.

Toran-Allerand CD, Singh M, Sétáló Jr G (1999) Novel mechanisms of estrogen action in the brain: new players in an old story. Front Neuroendocrinol 20:97-121.

Toran-Allerand CD, Guan X, MacLusky NJ, Horvath TL, Diano S, Singh M, Connolly Jr ES, Nethrapalli IS, Tinnikov AA (2002) ER-X: a novel, plasma membrane-associated, putative estrogen receptor that is regulated during development and after ischemic brain injury. J Neurosci 22:8391-8401.

Wehrenberg U, Prange-Kiel J, Rune GM (2001) Steroidogenic factor-1 expression in marmoset and rat hippocampus: co-localization with StAR and aromatase. J Neurochem 76:1879-1886.

Woolley CS, McEwen BS (1992) Estradiol mediates fluctuation in hippocampal synapse density during the estrous cycle in the adult rat. J Neurosci 12:2549-2554.

Woolley CS, McEwen BS (1993) Roles of estradiol and progesterone in regulation of hippocampal spine density during the estrous cycle in the rat. J Comp Neurol 336:293-306.

Woolley CS, Gould E, Frankfurt M, McEwen BS (1990) Naturally occurring fluctuation in dendritic spine density of adult hippocampal pyramidal neurons. J Neurosci 10:4035-4039.

Woolley CS, Wenzel HJ, Schwarzkroin PA (1996) Estradiol increases the frequency of multiple synapse boutons in the hippocampal CA1 region of the adult female rat. J Comp Neurol 373:108-117.

Yankova M, Hart SA, Woolley CS (2001) Estrogen increases synaptic connectivity between single presynaptic inputs and multiple postsynaptic CA1 pyramidal cells: a serial electron microscopic study. Proc Natl Acad Sci USA 98:3525-3530.

Yokomaku D, Numakawa T, Numakawa Y, Suzuki S, Matsumoto T, Adachi N, Nishio C, Taguchi T, Hatanaka H (2003) Estrogen enhances depolarization-induced glutamate relaease through activation of phosphatidylinositol 3-kinase and mitogen-activated protein kinase in cultured hippocampal neurons. Mol Endocrinol 17:831-844. 